\title{
Adding eltrombopag to immunosuppression: the importance of predicting outcome
}

\author{
André Tichelli, ${ }^{1,2}$ Régis Peffault de Latour, ${ }^{2,3}$ Carlo Dufour ${ }^{2,4}$ and Alicia Rovó ${ }^{2,5}$
}

\begin{abstract}
${ }^{1}$ Hematology, University Hospital, Basel, Switzerland; ${ }^{2}$ Severe Aplastic Anemia Working Party of the European Group for Blood and Marrow Transplantation, Leiden, the Netherlands; ${ }^{3}$ French Reference Center for Aplastic Anemia and PNH, BMT Unit, Saint-Louis Hospital. - AP-HP, Université de Paris, Paris, France; ${ }^{4}$ Hemato-Oncology and Stem Cell Transplantation Pole, G. Gaslini, Research Children's Hospital (IRCCS), Genova, Italy and 'Department of Hematology and Central Hematology Laboratory, Inselspital, Bern University Hospital, University of Bern, Bern, Switzerland
\end{abstract}

E-mail: ANDRÉ TICHELLI - andre.tichelli@gmail.com

doi:10.3324/haematol.2021.278761

S evere aplastic anemia (SAA) can be successfully treated today, with either allogeneic hematopoietic cell transplantation (HCT) or standard immunosuppressive therapy (IST) with horse antithymocyte globulin (hATG) and cyclosporine. The choice of one of these treatments is usually based on the availability of a matched sibling donor and the age of the patient. Despite good survival rates for both forms of treatment, there are relevant differences with respect to the long-term outcome. Thus, patients undergoing HCT are at risk of early complications mainly related to the toxicity of the preparative regimen, infections and acute graft-versus-host disease (GvHD), while in the long-term, they are prone to develop chronic GvHD. In contrast, patients treated with IST often do not have a complete recovery of blood counts, remaining with a certain degree of cytopenia, and are exposed to a series of risks including relapse of the underlying disease, development of a clinical paroxysmal nocturnal hemoglobinuria as well as late malignant clonal complications, such as myelodysplastic syndrome or acute myeloid leukemia. A major challenge of treating SAA patients is the identification of pretreatment factors predicting response, other than just age and donor availability, which could help to make the best therapeutic decision. A group from the National Institutes of Health contributed in this regard, showing in a previous study that pretreatment peripheral blood values were able to predict response of SAA patients treated with hATG and cyclosporine. ${ }^{1}$ More recently, eltrombopag, a thrombopoietin-receptor agonist added to standard IST, has been shown to improve the rate of response even for SAA patients, refractory to standard IST. ${ }^{2,3}$ Although a randomized comparison to standard immunosuppression is warranted and should be available soon (RACE; ClinicalTrials.gov number, NCT02099747; https://clinicaltrials.gov/ct2/show/NCT02099747, the use of a combination of hATG, cyclosporine and eltrombopag is tending to supplant standard IST for SAA and factors predicting response need to be confirmed with this triple combination.

In this issue of Haematologica, Zaimoku et al. show that baseline blood counts, such as reticulocyte, neutrophil, and lymphocyte counts, and thrombopoietin level, which are surrogates of residual hematopoiesis, remain the best predictors of response at 6 months after treatment with hATG, cyclosporine and eltrombopag. ${ }^{4}$ Interestingly, when compared to IST with hATG and cyclosporine alone, adding eltrombopag was predictive of response at 6 months, even with reticulocyte baseline counts below $30 \times 10^{9} / \mathrm{L}$ (between 10 and $30 \times 10^{9} / \mathrm{L}$ ), suggesting that the addition of eltrombopag to IST could increase the response rate also for SAA patients with less residual hematopoiesis. Furthermore, the authors suggested an age-dependent association between response and absolute lymphocyte counts, demonstrating that lower absolute lymphocyte counts in children under 10 years of age were predictive of complete response, while for older children and adults the reverse was the case.

Despite overall good survival after IST, event-free survival remains largely unsatisfactory. In a randomized study on IST with or without granulocyte colony-stimulating factor, the overall survival at 15 years was $60 \%$, but the event-free survival was as low as $24 \%$. This divergence was even more pronounced in patients 30 years of age or younger, who had an overall survival of $89 \%$ but an event-free survival of only $27 \%$ (Figure $1 \mathrm{~A})^{5}$, meaning that children and young adults treated with IST, despite excellent overall survival, still have a substantial number of complications later in life. After HCT, long-term follow-up shows greater coherence between overall survival and event-free survival, even when unrelated donors have been used (Figure 1B, C). ${ }^{6,7}$ A retrospective study of a selected cohort of pediatric patients with SAA demonstrated that upfront unrelated HCT produced similar results to those of matched sibling donor HCT and better than those of matched unrelated donor HCT after failure of IST. ${ }^{8}$ These data raise the question of whether upfrontunrelated donor HCT could be a first-line option in selected pediatric or young adult patients who lack a matched sibling donor and are at risk of refractoriness to IST. The broader the therapeutic options, the more important factors predicting response and outcome of IST become.

Event-free survival in patients treated with IST is mainly influenced by early deaths due to infections, refractoriness to treatment, relapse, the development of clinical paroxysmal nocturnal hemoglobinuria or transformation into a clonal myeloid neoplasm. Predicting the outcome of IST in SAA does, therefore, include three relevant issues: prediction of immediate response to treatment (affected by early infections and refractoriness to treatment), prediction of a robust and sustainable response in the long-term (affected by relapse of SAA), and prediction of the outcome in respect to late hematologic complications (affected by clonal malignant myeloid disease and clinical paroxysmal nocturnal hemoglobinuria). In a convincing manner, Zaimoku et al. determined factors predicting response to treatment at 6 months. Predicting events occurring after 6 months of IST would also be important in SAA patients, but this remains an unmet need in the field. In fact, identification of risk factors for relapse, development of paroxysmal nocturnal hemoglobinuria or transformation into 
malignant clonal hematopoietic diseases, which are relevant events affecting event-free survival after 6 months, would contribute considerably to predicting long-term outcomes.

The age-dependent association between response and absolute lymphocyte counts in the study by Zaimoku et al. is an interesting but not fully understood finding. The question of why lower absolute lymphocyte counts in children below 10 years predict complete response, while the reverse is true in older children and adults remains unanswered. A confirmatory study would be needed to validate the conflicting results. These paradoxical results remind us of another study in which pediatric patients with very SAA treated with IST showed unexpectedly

\section{A}

OS and EFS after IST in SAA patients $\leq \mathbf{3 0}$ years

B
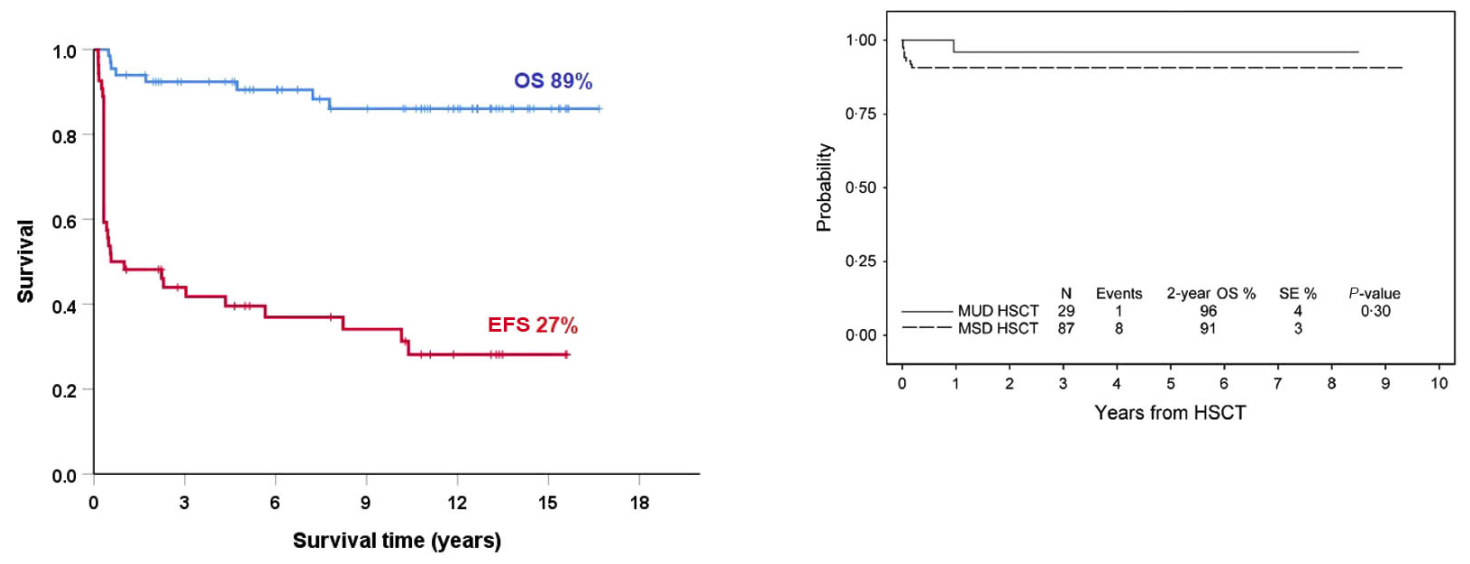

C

EFS after HCT in children with SAA

\section{D}

Treatment algorythm of SAA (adapted from EBMT
Handbook ; 2019)
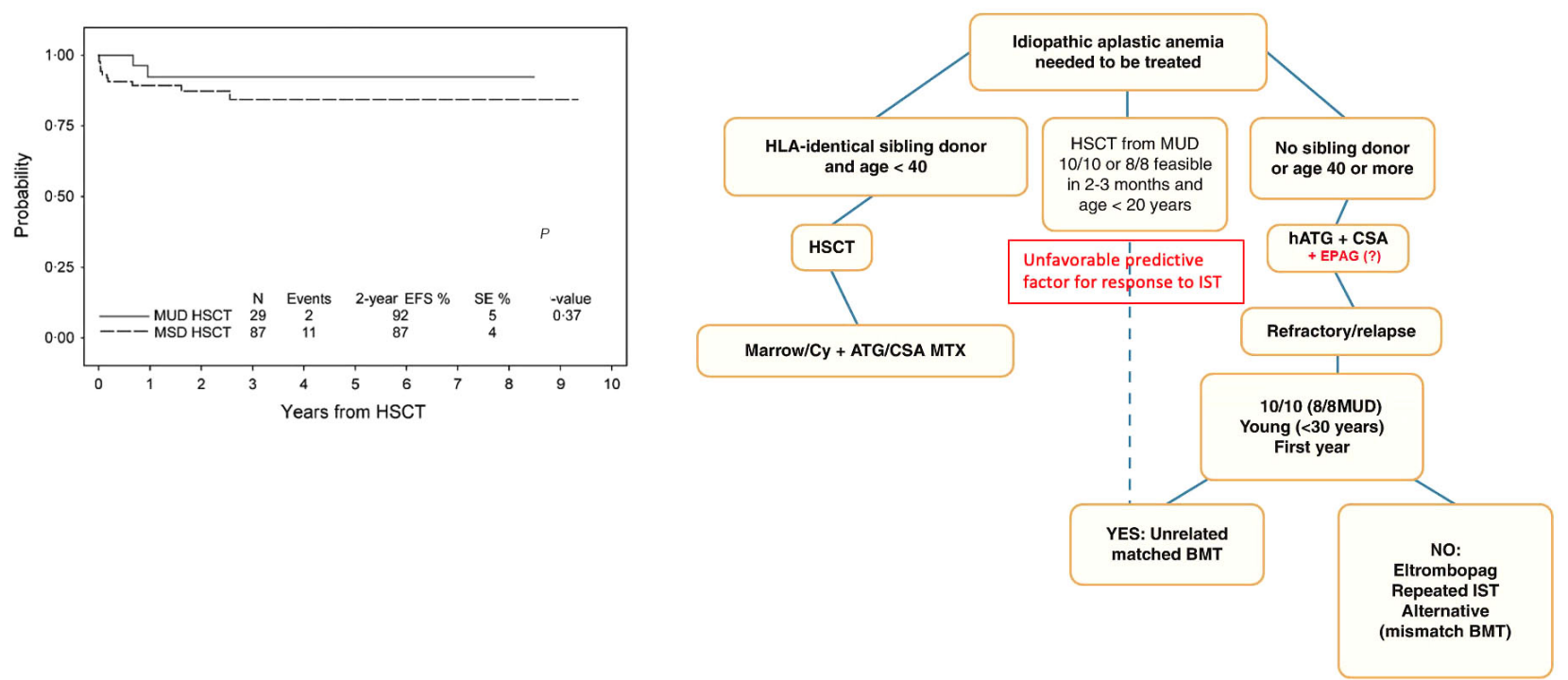

Figure 1. Survival outcomes of patients with severe aplastic anemia treated with immunosuppressive therapy or hematopoietic stem cell transplantation and a treatment algorithm. (A) Comparison of overall survival (OS) and event-free survival (EFS) of patients with severe aplastic anemia (SAA) 30 years of age and younger treated for SAA with immunosuppressive therapy (IST) with or without granulocyte colony-stimulating factor (from unpublished data of the dataset of a randomized study $^{5}$ ). Events were defined as refractoriness to first IST, relapse of SAA, development of clinical paroxysmal nocturnal hemoglobinuria or transformation into a myelodysplastic syndrome or acute leukemia. OS at 15 years is $89 \%$ and EFS $27 \%$ There is a great divergence between OS and EFS, meaning that children and young adults treated with IST, despite an excellent overall survival, still have a substantial number of complications later in life. These patients could, therefore, be considered as candidates for an alternative donor hematopoietic cell transplantation (HCT) if no matched sibling donor is available. (B, C) Survival outcomes in terms of OS (B) and EFS (C) following upfront-unrelated HCT in children with SAA. The divergence of OS and EFS is much less in patients treated with HCT than in those treated with IST (Figure from Dufour $\mathrm{C}$ et al. ${ }^{8}$ ). (D) The algorithm for first-line treatment adapted from the recommendations of the European Blood and Marrow Transplant Severe Aplastic Anemia Working Party (SAA-WP). ${ }^{10}$ Treatment with eltrombopag has been added in red, since it is not yet officially in the guideline. According to the recommendations of the SAA-WP, patients $<20$ years) could be considered for first-line HCT with a matched unrelated donor (MUD) if the donor is available within 2 to 3 months. The pretreatment predictive values discussed in the study by Zaimoku et al. ${ }^{4}$ could be helpful for decision-making. Indeed, patients with factors predicting an unfavorable response to IST could be the patients considered for first-line transplantation from a MUD. HSCT: hematopoietic stem cell transplantation; SE: standard error; MSD: matched sibling donor; ATG: antithymocyte globulin; CSA: cyclosporine A; MTX: methotrexate; hATG: horse antithymocyte globulin; EPAG: eltrombopag; BMT: bone marrow transplantation. 
better response to treatment and overall survival than children with less severe SAA. ${ }^{9}$ Unfortunately, baseline lymphocyte counts in that study were not available. We could hypothesize that similar mechanisms could explain discrepant results between young children and adult patients.

The data in the present study could also become a supportive tool to approach children and young adults with SAA who lack a matched sibling donor. The management of newly diagnosed pediatric and young adult SAA patients could be based on the following algorithm (Figure 1D): when baseline blood values are predictive of a unsatisfactory response to IST combined with eltrombopag, and the chance of rapidly finding a suitable alternative donor exists, upfront first-line HCT with this alternative donor should be taken in serious consideration. Future research in this field should be directed at identifying factors predicting long-term outcome, which would bring more precise information to adapt up-front treatment strategies.

\section{Disclosures}

No conflicts of interest to disclose.

\section{Contributions}

$A T, R P L, C D$ and $A R$ wrote the mansucript.

\section{References}

1. Scheinberg P, Wu CO, Nunez O, Young NS. Predicting response to immunosuppressive therapy and survival in severe aplastic anaemia.
Br J Haematol. 2009;144(2):206-216.

2. Assi R, Garcia-Manero G, Ravandi F, et al. Addition of eltrombopag to immunosuppressive therapy in patients with newly diagnosed aplastic anemia. Cancer. 2018;124(21):4192-4201.

3. Townsley DM, Scheinberg P, Winkler T, et al. Eltrombopag added to standard immunosuppression for aplastic anemia. N Engl J Med. 2017;376(16):1540-1550.

4. Zaimoku Y, Patel BA, Shalhoub R, et al. Predicting response of severe aplastic anemia to immunosuppression combined with eltrombopag. Haematologica. 2021;107(1):126-133.

5. Tichelli A, de Latour RP, Passweg J, et al. Long-term outcome of a randomized controlled study in patients with newly diagnosed severe aplastic anemia treated with antithymocyte globulin and cyclosporine, with or without granulocyte colony-stimulating factor: a Severe Aplastic Anemia Working Party trial from the European Group of Blood and Marrow Transplantation. Haematologica. 2020;105(5):1223-1231.

6. Yang S, Yuan X, Ma R, et al. Comparison of outcomes of frontline immunosuppressive therapy and frontline haploidentical hematopoietic stem cell transplantation for children with severe aplastic anemia who lack an HLA-matched sibling donor. Biol Blood Marrow Transplant. 2019;25(5):975-980.

7. Dufour C, Pillon M, Passweg J, et al. Outcome of aplastic anemia in adolescence: a survey of the Severe Aplastic Anemia Working Party of the European Group for Blood and Marrow Transplantation. Haematologica. 2014;99(10):1574-1581.

8. Dufour C, Veys P, Carraro E, et al. Similar outcome of upfront-unrelated and matched sibling stem cell transplantation in idiopathic paediatric aplastic anaemia. A study on behalf of the UK Paediatric BMT Working Party, Paediatric Diseases Working Party and Severe Aplastic Anaemia Working Party of EBMT. Br J Haematol. 2015;171(4):585-594.

9. Fuhrer M, Rampf U, Baumann I, et al. Immunosuppressive therapy for aplastic anemia in children: a more severe disease predicts better survival. Blood. 2005;106(6):2102-2104.

10. de Latour RP, Risitano A, Dufour C. Severe Aplastic Anemia and PNH. In: Carreras E, Dufour C, Mohty M, Kroger N, editors. The EBMT Handbook: Hematopoietic Stem Cell Transplantation and Cellular Therapies. 7th edition. Cham (CH): 2019. p. 579-585. 